\title{
Homogenization of acoustic metamaterials of Helmholtz resonators in fluid
}

\author{
Xinhua $\mathrm{Hu}^{*}$ and Kai-Ming $\mathrm{Ho}$ \\ Ames Laboratory and Department of Physics and Astronomy, Iowa State University, Ames, Iowa 50011, USA \\ C. T. Chan \\ Department of Physics, Hong Kong University of Science and Technology, Clear Water Bay, Kowloon, \\ Hong Kong, People's Republic of China \\ Jian $\mathrm{Zi}$ \\ Department of Physics and Surface Physics Laboratory, Fudan University, Shanghai 200433, People's Republic of China
}

(Received 6 April 2008; published 1 May 2008)

\begin{abstract}
By using a two-step homogenization approach, we derive analytical formulas of effective mass density $\rho_{e}$ and effective bulk modulus $B_{e}$ for two- and three-dimensional acoustic metamaterials of Helmholtz resonators (HRs) in fluid. A negative $B_{e}$ is found at certain frequencies due to the monopolar resonance, leading to a low-frequency acoustic band gap. A unified picture is presented for metamaterials of HRs and three-component metamaterials of negative $\rho_{e}$. Our work supports recent observations in a one-dimensional array of HRs [N. Fang et al., Nat. Mater. 5, 452 (2006)] and presents important high-dimensional extensions for exploring more fascinating phenomena.
\end{abstract}

DOI: $10.1103 /$ PhysRevB.77.172301

PACS number(s): 42.25.Bs, 41.20.Jb, 46.40.-f, 62.30.+d

Recently, acoustic and/or elastic metamaterials (artificial structured materials ${ }^{1,2}$ ) have received considerable interest due to their exotic acoustic and/or elastic properties. ${ }^{3-17}$ In particular, their effective mass density and modulus can be singly or simultaneously negative, ${ }^{3-13}$ allowing intriguing phenomena for sound waves such as low-frequency band gaps, ${ }^{3-10}$ negative refraction, and superlensing. $.12-14$

Negative effective mass density or modulus can occur at certain frequencies if an appropriate resonance is included into the structures. ${ }^{3-13}$ A famous example is the threecomponent phononic crystal with locally resonant structures, ${ }^{3}$ which exhibits a negative effective mass density $\rho_{e}$ due to a dipolar resonance. ${ }^{4,5}$ Very recently, a lowfrequency band gap was demonstrated in a one-dimensional (1D) array of Helmholtz resonators (HRs). ${ }^{6}$ By using further retrieval analysis of wave-scattering coefficients, ${ }^{7-9}$ a negative effective bulk modulus $B_{e}$ was confirmed in the 1D metamaterials of HRs. ${ }^{9}$ Unlike other designs for negative $B_{e}$ using air bubbles or soft-rubber spheres, ${ }^{12,13}$ this metamaterial consists of a rigid body but still possesses a sizable working frequency range. However, high-dimensional metamaterials of HRs, which are essential for more fascinating phenomena, ${ }^{6,14,15}$ remain unexplored.

In this Brief Report, we study two-dimensional (2D) and three-dimensional (3D) acoustic metamaterials consisting of cylindrical and spherical HRs in fluid (such as water or air), respectively. By using a two-step homogenization approach, we can derive analytical formulas for the $\rho_{e}$ and $B_{e}$ within coherent-potential approximation (CPA). ${ }^{18}$ The accuracy of these formulas is confirmed by accurate retrieval results using multiple-scattering techniques. We show that our metamaterials of HRs can exhibit a negative $B_{e}$ at certain frequencies due to a monopolar resonance. Systematic analyses are done for the frequency range of negative $B_{e}$ and the low-frequency-limit behavior. Based on our derivations, a unified picture is presented for metamaterials of HRs and three-component metamaterials of negative $\rho_{e}$.
Our HRs are cylindrical and/or spherical rigid shells with uniformly distributed slits and/or holes, as shown in Fig. 1(c). The inner and outer radii of the shells are $r_{2}$ and $r_{1}$, respectively. The background fluid has a mass density $\rho_{0}$ and bulk modulus $B_{0}$. Our HR becomes a conventional HR if only a single slit and/or hole exists in the shell. It has been shown that for acoustic waves of long wavelength $\left(\lambda_{0}\right)$, a rigid-body slab with small slits and/or holes (with a size $\Delta$ $<d / 4$ and period $d<\lambda_{0} / 4$ ) is effectively equivalent to a uniform slab of a thickness $h / n$, density $\rho_{0} n^{2}$, and bulk modulus $B_{0}$, where $h$ is the thickness of the rigid-body slab, $n=1 / f$, and $f$ is the filling ratio of slits and/or holes $(f=d / \Delta$ for slits). ${ }^{17}$ In the following, we will use this conclusion and

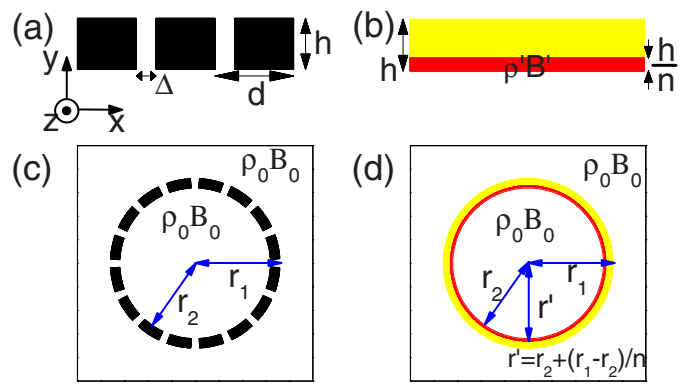

FIG. 1. (Color online) (a) A rigid-body slab with periodic slits (holes) and (b) its equivalence of a two-layer slab. The slabs have a thickness $h$ and are extending in the $X-Z$ plane. The slits and/or holes have a size $\Delta$, period $d$, and filling ratio $f(f=d / \Delta$ for slits). The top layer has a thickness $h-h / n$ but the same fields at their two surfaces, and the bottom layer has a thickness $h / n$, density $\rho^{\prime}$ $=\rho_{0} n^{2}$ with $n=1 / f$, and modulus $B^{\prime}=B_{0}$. The background fluid has a density $\rho_{0}$ and modulus $B_{0}$. (c) A 2D (3D) HR of rigid cylindrical (spherical) shell with uniformly distributed slits (holes) and (d) its equivalence of a two-layer shell made of the slabs in (a) and (b), respectively. 


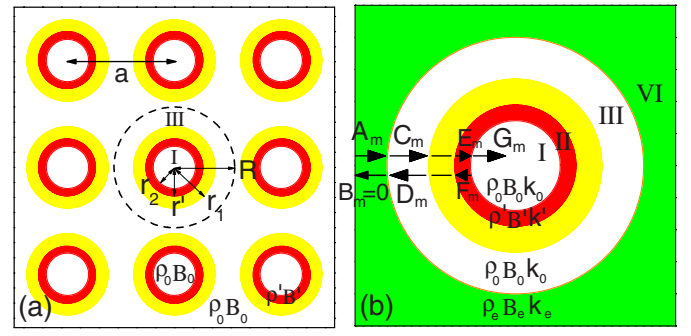

FIG. 2. (Color online) (a) A 2D (3D) acoustic metamaterial consisting of a square (simple cubic) lattice of the cylindrical (spherical) shells in Fig. 1(d) and (b) a coated shell in an effective medium of $\left(\rho_{e}, B_{e}\right)$.

replace the wall of the HR by its equivalence of a two-layer shell, ${ }^{19}$ where the inner layer has a thickness $\left(r_{1}-r_{2}\right) / n$, density $\rho^{\prime}=\rho_{0} n^{2}$, and modulus $B^{\prime}=B_{0}$, and the outer layer has a thickness $\left(r_{1}-r_{2}\right)(1-1 / n)$ but the same fields at its two surfaces. Here, we introduce the outer layer to make the total wall thickness unchanged.

We first consider a 2D metamaterial consisting of a square array of above cylindrical HRs in fluid, as shown in Fig. 2(a). The filling ratio of HRs is $f_{s}=\pi r_{1}^{2} / a^{2}$, where $a$ is the lattice constant. Set $\mathbf{r}=(x, y)=(r, \theta)$ in the plane perpendicular to the axes of HRs. For harmonic acoustic waves with angular frequency $\omega$, the pressure field $P(\mathbf{r}) e^{i \omega t}$ obeys the two-dimensional Helmholtz equation,

$$
\left(\nabla^{2}+k^{2}\right) P=0
$$

which is subjected to continuities of $P$ and $\frac{1}{\rho} \partial P / \partial r$ at the layer interfaces of each HR (set $\mathbf{r}=0$ at the center of HR). The wave numbers in the background and inner walls of HRs are given by $k_{0}=\omega \sqrt{\rho_{0} / B_{0}}$ and $k^{\prime}=\omega \sqrt{\rho^{\prime} / B^{\prime}}=n k_{0}$, respectively.

Then, we derive analytical formulas for the $\rho_{e}$ and $B_{e}$ of HRs by the CPA method. ${ }^{18}$ We consider a "circular unit cell" (coated HR) of radius $R=r_{1} / \sqrt{f_{s}}$ and replace the metamaterial outside by a uniform effective medium of $\rho_{e}, B_{e}$, and $k_{e}=\omega \sqrt{\rho_{e} / B_{e}}$ [see Fig. 2(b)]. The pressure field $P$ can be decomposed into incident and scattering cylindrical waves [represented by Bessel $\left(J_{m}\right)$ and Hankel $\left(H_{m}\right)$ functions, respectively]: $\quad P^{\mathrm{I}}=\Sigma_{m} G_{m} J_{m}\left(k_{0} r\right) e^{i m \theta} \quad$ when $\quad r<r_{2}, \quad P^{\mathrm{II}}$ $=\Sigma_{m}\left[E_{m} J_{m}\left(k^{\prime} r\right)+F_{m} H_{m}\left(k^{\prime} r\right)\right] e^{i m \theta}$ when $r_{2}<r<r^{\prime} \equiv r_{2}+\left(r_{1}\right.$ $\left.-r_{2}\right) / n, \quad P^{\mathrm{III}}=\sum_{m}\left[C_{m} J_{m}\left(k_{0} r\right)+D_{m} H_{m}\left(k_{0} r\right)\right] e^{i m \theta}$ when $r_{1}<r$ $<R$, and $P^{\mathrm{VI}}=\Sigma_{m}\left[A_{m} J_{m}\left(k_{e} r\right)+B_{m} H_{m}\left(k_{e} r\right)\right] e^{i m \theta}$ when $r>R$. For the outer layer of HR, the fields will be the same at its two surfaces so that $P^{\mathrm{II}}\left(r^{\prime}\right)=P^{\mathrm{III}}\left(r_{1}\right)$ and $\frac{1}{\rho^{\prime}} \frac{\partial P^{\mathrm{II}}\left(r^{\prime}\right)}{\partial r}=\frac{1}{\rho_{0}} \frac{\partial P^{\mathrm{II}}\left(r_{1}\right)}{\partial r}$.

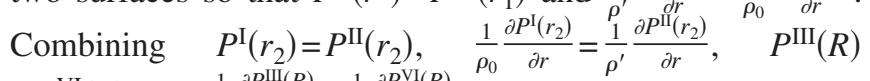
$=P^{\mathrm{VI}}(R)$, and $\frac{1}{\rho_{0}} \frac{\partial P^{\mathrm{II}}(R)}{\partial r}=\frac{1}{\rho_{e}} \frac{\partial P^{\mathrm{VI}}(R)}{\partial r}$, the $m$ th order scattering coefficient $B_{m} / A_{m}$ of the coated HR can be obtained and $B_{m} / A_{m}=0$ (which "defines" the effective medium) when

$$
\begin{gathered}
-\frac{\frac{1}{\rho_{0}} k_{0} J_{m}^{\prime}\left(k_{0} R\right)-J_{m}\left(k_{0} R\right) \frac{1}{\rho_{e}} k_{e} J_{m}^{\prime}\left(k_{e} R\right) / J_{m}\left(k_{e} R\right)}{\frac{1}{\rho_{0}} k_{0} H_{m}^{\prime}\left(k_{0} R\right)-H_{m}\left(k_{0} R\right) \frac{1}{\rho_{e}} k_{e} J_{m}^{\prime}\left(k_{e} R\right) / J_{m}\left(k_{e} R\right)} \\
=-\frac{\frac{1}{\rho_{0}} k_{0} J_{m}^{\prime}\left(k_{0} r_{1}\right)-J_{m}\left(k_{0} r_{1}\right) U_{m}}{\frac{1}{\rho_{0}} k_{0} H_{m}^{\prime}\left(k_{0} r_{1}\right)-H_{m}\left(k_{0} r_{1}\right) U_{m}},
\end{gathered}
$$

where

$$
U_{m}=\frac{1}{\rho^{\prime}} k^{\prime} \frac{J_{m}^{\prime}\left(k^{\prime} r^{\prime}\right)+H_{m}^{\prime}\left(k^{\prime} r^{\prime}\right) P_{m}}{J_{m}\left(k^{\prime} r^{\prime}\right)+H_{m}\left(k^{\prime} r^{\prime}\right) P_{m}}
$$

and

$$
P_{m}=-\frac{\frac{1}{\rho^{\prime}} k^{\prime} J_{m}^{\prime}\left(k^{\prime} r_{2}\right)-J_{m}\left(k^{\prime} r_{2}\right) \frac{1}{\rho_{0}} k_{0} J_{m}^{\prime}\left(k_{0} r_{2}\right) / J_{m}\left(k_{0} r_{2}\right)}{\frac{1}{\rho^{\prime}} k^{\prime} H_{m}^{\prime}\left(k^{\prime} r_{2}\right)-H_{m}\left(k^{\prime} r_{2}\right) \frac{1}{\rho_{0}} k_{0} J_{m}^{\prime}\left(k_{0} r_{2}\right) / J_{m}\left(k_{0} r_{2}\right)} .
$$

We note that the first and second terms in Eq. (2) and $P_{m}$ are just the $m$ th order scattering coefficients of a cylinder with radius $R, \rho_{e}$, and $B_{e}$ in a matrix of $\rho_{0}, B_{0}$, the $\mathrm{HR}$, and the core cylinder, respectively. If we only consider the scattering of cylindrical waves of the two lowest orders $m=0$ and $1, \rho_{e}$ and $B_{e}$ can be numerically calculated after solving $\frac{1}{\rho_{e}} k_{e} J_{0}^{\prime}\left(k_{e} R\right) / J_{0}\left(k_{e} R\right)$ and $\frac{1}{\rho_{e}} k_{e} J_{1}^{\prime}\left(k_{e} R\right) / J_{1}\left(k_{e} R\right)$ from Eq. (2).

Under the approximation of long wavelength $\left[k_{0} a, k_{e} a\right.$ $\ll 1$, thus $\left.k^{\prime}\left(r^{\prime}-r_{2}\right)=k_{0}\left(r_{1}-r_{2}\right) \ll 1\right]$ and small slit size $(n$ $=1 / f \gg 1)$, Eq. (2) becomes ${ }^{20}$

$$
\begin{aligned}
& \frac{1}{B_{e}}=\frac{1}{B_{0}}\left(1-f_{s}+\frac{f_{s} r_{2} / r_{1}}{1-\omega^{2} / \omega_{R}^{2}}\right) \quad \text { for } m=0, \\
& \rho_{e}=\rho_{0}\left[1+f_{s} /(L-1)\right] /\left(1-f_{s}\right) \quad \text { for } m=1,
\end{aligned}
$$

where $L=2$ in the $2 \mathrm{D}$ case and the resonant frequency is given by

$$
\omega_{R}=v_{0} \sqrt{L /\left[n r_{2}\left(r_{1}-r_{2}\right)\right]} .
$$

Here, $v_{0}=\sqrt{B_{0} / \rho_{0}}$ is the sound velocity in the background fluid. If only a single slit exists in the wall of HR ( $n$ $\approx 2 \pi r_{2} / \Delta$, where $\Delta$ is the slit width), Eq. (5) will become the conventional HR frequency in 2D, namely, $\omega_{R}$ $=v_{0} \sqrt{\Delta /\left[\pi r_{2}^{2}\left(r_{1}-r_{2}\right)\right]} \cdot{ }^{21}$ For the case without slits $(n \rightarrow \infty)$, $\omega_{R}$ will approach zero and Eq. (3) will reduce to the formula of $B_{e}$ for rigid rods in fluid [where $\rho_{e}$ is still given by Eq. (4)] ${ }^{16}$ namely,

$$
B_{e}=B_{0} /\left(1-f_{s}\right) .
$$

From Eq. (3), we can see that the $B_{e}$ of HRs depends on frequency. At the low-frequency limit $(\omega \rightarrow 0)$, the effective bulk modulus is given by

$$
B_{e}=B_{0} /\left(1-f_{s}+f_{s} r_{2} / r_{1}\right) .
$$




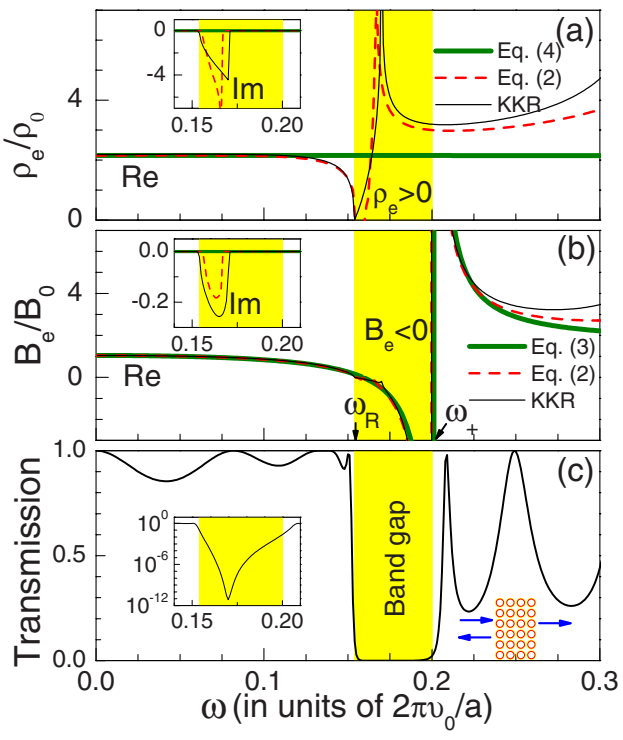

FIG. 3. (Color online) (a) The effective mass density and (b) bulk modulus for the 2D acoustic metamaterial in Fig. 2(a), and (c) the transmission spectrum for normal incidence of a plane wave on a four-layer metamaterial. The parameters are $r_{1}=0.36 a, r_{2}=0.32 a$, and $f=6.39 \times 10^{-3}$ (thus $n=156.5, \quad r^{\prime}=0.320256 a, \quad \rho^{\prime}=2.45$ $\times 10^{4} \rho_{0}$, and $\left.B^{\prime}=B_{0}\right)$.

Here, $B_{e}$ will be zero at the resonant frequency $\omega_{R}$. For a frequency range above $\omega_{R}\left(\omega_{R}<\omega<\omega_{+}\right), B_{e}$ becomes negative, where the upper frequency edge is given by

$$
\omega_{+}=\omega_{R} \sqrt{1+f_{s} r_{2} /\left[r_{1}\left(1-f_{s}\right)\right]} .
$$

Since the effective density $\rho_{e}$ will always be positive [by Eq. (4)], an acoustic band gap (with an imaginary $k_{e}$ ) will be formed in the frequency range with negative $B_{e}$.

Similar CPA derivations can also be done for 3D metamaterials consisting of HRs of spherical shells with uniformly distributed holes. In 3D, ${ }^{22}$ the Bessel $\left(J_{m}\right)$ and Hankel $\left(H_{m}\right)$ functions in Eq. (2) should be replaced by spherical Bessel $\left(j_{m}\right)$ and Hankel $\left(h_{m}\right)$ functions, respectively. Under similar approximation, Eqs. (3)-(8) will also be obtained where $f_{s}$ becomes the volume ratio of spherical HRs $\left(f_{s}=\frac{4}{3} \pi r_{1}^{3} / a^{3}\right.$ in a simple cubic lattice) and $L=3$ in the $3 \mathrm{D}$ case. For HRs of single hole ( $n \approx 4 \pi r_{2}^{2} / S_{h}$, where $S_{h}$ is the area of the hole), Eq. (5) will become the conventional formula of HR frequency, namely, $\omega_{R}=v_{0} \sqrt{S_{h} /\left[\frac{4}{3} \pi r_{2}^{3}\left(r_{1}-r_{2}\right)\right]} .{ }^{21}$ When no holes exist in the shells $(n \rightarrow \infty), \omega_{R}$ will approach zero and Eqs. (4) and (6) can be obtained for rigid spheres in fluid.

To check the validity of the above analytic formulas, we apply the multiple-scattering or Korringa-Kohn-Rostoker (KKR) method (including high-order cylindrical waves) ${ }^{23}$ to simulate normal incidence of a plane wave on a slab of above 2D acoustic metamaterial [in Fig. 2(a)]. Then, the effective parameters $\left(B_{e}, \rho_{e}\right)$ can be retrieved from the transmission and reflection coefficients. ${ }^{7-9}$ The retrieving is easy for a one-layer slab but the results will be the same for more layers. In Fig. 3, we show the accurate KKR results of $\left(B_{e}, \rho_{e}\right)$ and those from Eqs. (2)-(4) for a square lattice of cylindrical HRs in fluid where $r_{1}=0.36 a, r_{2}=0.32 a$, and
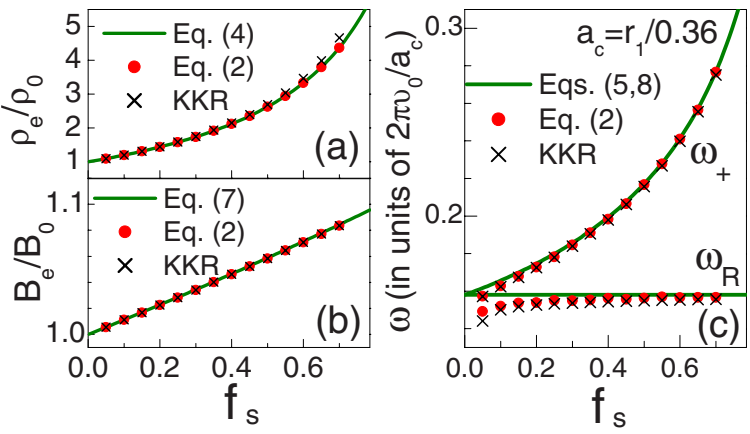

FIG. 4. (Color online) (a) The effective mass density and (b) bulk modulus when $\omega r_{1} / v_{0} \rightarrow 0$, and (c) the edges of the frequency range with a negative $B_{e}$ as functions of the filling fraction $f_{s}$ $\equiv \pi r_{1}^{2} / a^{2}$ for the 2D acoustic metamaterials in Fig. 2(a). The parameters of $r_{1} / r_{2}$ and $f$ are as in Fig. 3 .

$f=6.39 \times 10^{-3} \quad$ (thus, $n=156.5, \quad r^{\prime}=0.320256 a, \quad \rho^{\prime}=2.45$ $\times 10^{4} \rho_{0}$, and $\left.B^{\prime}=B_{0}\right)$. A frequency range $\left[\widetilde{\omega} \equiv \omega_{0} a /\left(2 \pi v_{0}\right)\right.$ $\in(0.154,0.2)]$ with a negative $B_{e}$ and positive $\rho_{e}$ can be found above the resonant frequency. As a result, a lowfrequency stop band can be observed in the transmission spectrum for a metamaterial slab [see Fig. 3(c)]. We note that the transmission dip at $\widetilde{\omega}=0.170$ corresponds to a peak of $\rho_{e}=9.77-i 4.45$ (with $B_{e}=-0.194-i 0.09$ which will not bring a gain or loss; KKR results). This peak of $\rho_{e}$ cannot be captured by Eq. (4) with the long-wavelength approximation and it is usually understood as a result of band folding. However, we find that this feature can still be obtained by numerically solving Eq. (2) for the lowest 2 orders of cylindrical waves. For higher frequencies $(\tilde{\omega}>0.25)$, the scattering of highorder cylindrical waves will be important and more accurate KKR calculations are needed.

Figure 4 shows the $\rho_{e}$ and $B_{e}$ at the low-frequency limit $\left(\omega r_{1} / v_{0} \ll 1\right)$ and the frequency edges of negative $B_{e}$ with varying the filling fraction $f_{s}$ of HRs. The frequency range of negative $B_{e}$ is found wider with increasing the filling ratio of HRs. Excellent agreement is found between our analytical formulas and KKR results. Recently, a better impedance match and higher refractive index were found in 2D HRs than rigid rods by finite-difference time-domain simulations. ${ }^{7}$ This result was explained by a smaller $B_{e}$ of HRs $\left(B_{e}=B_{0}\right)$ than rods. We note that our new Eq. (7) can present a better understanding for this phenomenon. ${ }^{20}$

By now, we have shown that our metamaterials of HRs exhibit a negative effective bulk modulus at certain frequencies due to a monopolar $(m=0)$ resonance. For the HR, the fluid inside the heavy shell $\left(\rho^{\prime}=\rho_{0} n^{2} \gg \rho_{0}\right)$ will retain a cylindrical and/or spherical shape. However, its volume can still periodically vary ( since $B^{\prime}=B_{0}$ ) giving rise to the monopolar resonance. Strong monopolar resonances can also exist in air bubbles ${ }^{11,13}$ or soft-rubber spheres $^{12}$ in water, leading to a negative $B_{e} \cdot{ }^{12,13}$ Such monopolar resonances can still survive even when a solid matrix is used (e.g., watercoated air bubbles in solid). ${ }^{13}$ So if a solid-based structure with a negative $B_{e}$ is desired, fluid-coated HRs in a solid matrix can be similarly applied. By combining our HRs with the metamaterials of a negative $\rho_{e}$, a double negative metamaterial can be constructed, ${ }^{13}$ enabling negative refrac- 
tion and superlensing of acoustic waves. We note that our HRs can also exhibit an arbitrary positive $B_{e}\left[0<B_{e}\right.$ $<B_{0} /\left(1-f_{s}+f_{s} r_{2} / r_{1}\right)$ or $\left.B_{e}>B_{0} /\left(1-f_{s}\right)\right]$ but normal $\rho_{e}$ [by Eq. (4)] at certain frequencies $\left(\omega<\omega_{R}\right.$ or $\left.\omega>\omega_{+}\right)$. When an additional metamaterial with an anisotropic $\rho_{e}$ but normal $B_{e}$ is provided, a fascinating acoustic cloaking may also be realized. $^{15}$

A coated-cylinder and/or coated-sphere structure exists in our acoustic metamaterials of HRs and the locally resonant sonic materials with soft rubber. ${ }^{3}$ However, a negative effective mass density was found in the latter. ${ }^{4,5}$ To present a unified picture for these two metamaterials, we mathematically consider metamaterials of soft shells in fluid [by using $B^{\prime}=B_{0} / n^{2} \ll B_{0}$ and $\rho^{\prime}=\rho_{0}$ in Fig. 1(d)]. Under the longwavelength approximation $\left[k_{0} a, k_{e} a \ll 1\right.$, thus $\left.k^{\prime}\left(r^{\prime}-r_{2}\right) \ll 1\right]$, Eq. (2) becomes ${ }^{20}$

$$
\begin{gathered}
\frac{1}{B_{e}}=\frac{1}{B_{0}}\left[1+f_{s}(L n-1)\left(1-\frac{r_{2}}{r_{1}}\right)\right], \\
\frac{\rho_{e}-\rho_{0}}{\rho_{e}+\rho_{0}}=-f_{s}+f_{s} \frac{L r_{2} /\left(r_{1}+r_{2}\right)}{1-\omega^{2} / \omega_{R}^{\prime 2}},
\end{gathered}
$$

for $m=0$ and 1 , respectively, where the resonant frequency is given by

$$
\omega_{R}^{\prime}=v_{0} \sqrt{\left(L-1+r_{1} / r_{2}\right) /\left[n r_{1}\left(r_{1}-r_{2}\right)\right]} .
$$

From Eq. (10), a negative effective mass density can be seen due to a dipolar $(m=1)$ resonance, which agrees with recent explanations. ${ }^{4,5}$ For the soft shell, the fluid inside will retain the shape and volume and will vibrate in a certain direction, giving rise to the dipolar resonance.

In summary, we have proposed a two-step homogenization approach to derive analytical formulas of $\rho_{e}$ and $B_{e}$ for $2 \mathrm{D}$ and $3 \mathrm{D}$ acoustic metamaterials of HRs in fluid. A negative $B_{e}$ was found at certain frequencies due to the monopolar resonance, leading to a low-frequency acoustic band gap. A unified picture was also presented for metamaterials of HRs and three-component metamaterials of negative $\rho_{e}$. Our work supported recent observations in 1D array of HRs and presented important high-dimensional extensions for exploring more fascinating acoustic phenomena.

X.H. thanks Zhengyou Liu for communications. This work is supported by the Director for Energy Research, Office of Basic Energy Sciences. C.T.C. is supported by HK RGC under Grant No. 600305. J.Z. acknowledges support from the 973 Program and NSFC. The Ames Laboratory is operated for the U.S. Department of Energy by Iowa State University under Contract No. W-7405-ENG-82.

\footnotetext{
*xhhu@iastate.edu

${ }^{1}$ V. G. Veselago, Sov. Phys. Usp. 10, 509 (1968); J. B. Pendry, Phys. Rev. Lett. 85, 3966 (2000).

${ }^{2}$ For a recent review, see D. R. Smith, J. B. Pendry, and M. Wiltshire, Science 305, 788 (2004).

${ }^{3}$ Z. Liu et al., Science 289, 1734 (2000).

${ }^{4}$ Z. Liu, C. T. Chan, and P. Sheng, Phys. Rev. B 71, 014103 (2005).

${ }^{5}$ Y. Wu, Y. Lai, and Z. Q. Zhang, Phys. Rev. B 76, 205313 (2007).

${ }^{6}$ N. Fang et al., Nat. Mater. 5, 452 (2006).

${ }^{7}$ X. Hu, C. T. Chan, and J. Zi, Phys. Rev. E 71, 055601(R) (2005).

${ }^{8}$ V. Fokin, M. Ambati, C. Sun, and Xiang Zhang, Phys. Rev. B 76, 144302 (2007).

${ }^{9}$ Z. G. Wang et al., J. Phys.: Condens. Matter 20, 055209 (2008).

${ }^{10}$ Z. Hou, J. Liu, W. Kuang, Y. Liu, and S. Wu, Phys. Rev. E 75, 026608 (2007).

${ }^{11}$ M. Kafesaki, R. S. Penciu, and E. N. Economou, Phys. Rev. Lett. 84, 6050 (2000).

${ }^{12}$ J. Li and C. T. Chan, Phys. Rev. E 70, 055602(R) (2004).

${ }^{13}$ Y. Ding, Z. Liu, C. Qiu, and J. Shi, Phys. Rev. Lett. 99, 093904 (2007).

${ }^{14}$ M. Ambati, N. Fang, C. Sun, and X. Zhang, Phys. Rev. B 75, 195447 (2007).

${ }^{15}$ W. Milton, M. Briane, and J. R. Willis, New J. Phys. 8, 248
}

(2006); S. A. Cummer and D. Schurig, ibid. 9, 45 (2007); H. Chen and C. T. Chan, Appl. Phys. Lett. 91, 183518 (2007); S. A. Cummer, B. I. Popa, D. Schurig, D. R. Smith, J. Pendry, M. Rahm, and A. Starr, Phys. Rev. Lett. 100, 024301 (2008).

${ }^{16}$ X. Hu and C. T. Chan, Phys. Rev. Lett. 95, 154501 (2005); D. Torrent, A. Hakansson, F. Cervera, and J. Sanchez-Dehesa, ibid. 96, 204302 (2006); J. Mei, Z. Liu, W. Wen, and P. Sheng, ibid. 96, 024301 (2006).

${ }^{17}$ F. Cai, F. Liu, Z. He, and Z. Liu, Appl. Phys. Lett. 91, 203515 (2007); (to be published).

${ }^{18} \mathrm{P}$. Sheng, Introduction to Wave Scattering Localization and Mesoscopic Phenomena (Academic, New York, 1995).

${ }^{19}$ Based on the coordinate transformation method, the two-layer slab can be transformed to a one-layer slab with the same slab thickness. However, anisotropic effective parameters will be found. Here, we use this two-layer slab for convenience of analytical derivation.

${ }^{20}$ See EPAPS Document No. E-PRBMDO-77-015817 for derivation of Eqs. (3), (4), (9), and (10) and comparison of our formulas with FDTD results. For more information on EPAPS, see http://www.aip.org/pubservs/epaps.html

${ }^{21}$ L. E. Kinsler, Fundamentals of Acoustics (Wiley, New York, 1982).

${ }^{22}$ M. Kafesaki and E. N. Economou, Phys. Rev. B 60, 11993 (1999).

${ }^{23}$ L. C. Botten et al., J. Opt. Soc. Am. A 17, 2165 (2000). 